\title{
Probing the nucleation of iron in Earth's core using molecular dynamics simulations of supercooled liquids
}

\author{
Alfred J. Wilson $\odot,,^{1, *}$ Andrew M. Walker $\odot,{ }^{2,1}$ Dario Alfè $\odot,{ }^{3,4,5}$ and Christopher J. Davies $\oplus^{1}$ \\ ${ }^{1}$ School of Earth and Environment, University of Leeds, Woodhouse, Leeds LS2 9JT, United Kingdom \\ ${ }^{2}$ Department of Earth Sciences, University of Oxford, 3 South Parks Rd., Oxford OX1 3AN, United Kingdom \\ ${ }^{3}$ Department of Earth Sciences, Thomas Young Center, University College London, 5 Gower Place, London WC1E 6BS, United Kingdom \\ ${ }^{4}$ London Centre for Nanotechnology, Thomas Young Centre, University College London, 17-19 Gordon Street, \\ London WC1H OAH, United Kingdom \\ ${ }^{5}$ Dipartimento di Fisica Ettore Pancini, Universitá di Napoli Federico II, Monte S. Angelo, 80126 Napoli, Italy
}

(Received 28 April 2021; accepted 11 June 2021; published 23 June 2021)

\begin{abstract}
Classical nucleation theory describes the formation of the first solids from supercooled liquids and predicts an average waiting time for a system to freeze as it is cooled below the melting temperature. For systems at low to moderate undercooling, waiting times are too long for freezing to be observed via simulation. Here a system can be described by estimated thermodynamic properties, or by extrapolation from practical conditions where thermodynamic properties can be fit directly to simulations. In the case of crystallizing Earth's solid iron inner core, these thermodynamic parameters are not well known and waiting times from simulations must be extrapolated over approximately 60 orders of magnitude. In this work, we develop a new approach negating the need for freezing to be observed. We collect statistics on solidlike particles in molecular dynamic simulations of supercooled liquids at $320 \mathrm{GPa}$. This allows estimation of waiting times at temperatures closer to the melting point than is accessible to other techniques and without prior thermodynamic insight or assumption. Our method describes the behavior of nucleation at otherwise inaccessible conditions such that the nucleation of any system at small undercooling can be characterized alongside the thermodynamic quantities which define the first formed solids.
\end{abstract}

DOI: 10.1103/PhysRevB.103.214113

\section{INTRODUCTION}

In order for a pure liquid to freeze, it must cool significantly below its melting temperature $\left(T_{m}\right.$; e.g., [1]). This requirement is the result of an interface separating the two phases when a solid forms. While the solid is thermodynamically favored for temperatures below $T_{m}$, a solid-liquid interface remains unfavorable and so a commensurate difference between liquid and solid free energies is required. The phenomenon of supercooling is well studied in metallurgy and meteorology where precipitation is important (e.g., [2,3]). It also forms the basis of this work's motivation, the inner core nucleation paradox [4], where the cooling rate of the Earth's core cannot be reconciled with sufficient undercooling to have crystallized the seismically observed solid inner core. According to classical nucleation theory (CNT), for the inner core to have crystallized, undercooling on the order of $1000 \mathrm{~K}$ is apparently required. However, if this were the case, after the onset of crystallization all material below $T_{m}$ will freeze resulting in an inner core that is much larger than observed.

CNT (e.g., [1]) describes the nucleation rate (I) of solids in supercooled liquids via three components [Eq. (1)]: (1) A free energy associated with forming a nucleus $(\Delta G)$. (2) Boltzmann statistics defining the probability of atoms forming

\footnotetext{
*a.j.wilson1@leeds.ac.uk
}

a solidlike arrangement representing a nucleus. The stochastic nature of the nucleation process is represented here; a nucleus with a low probability of forming will correspond to a long average duration before such a configuration is randomly sampled, referred to as the waiting time $\left(\tau_{w}\right)$. (3) A density of available nucleation sites and rate at which atoms can be attached define a prefactor $\left(I_{0}\right)$ which scales the nucleation rate

$$
I=I_{0} \exp \left(\frac{-\Delta G}{k_{B} T}\right)
$$

$\Delta G$ is described in CNT by the interfacial energy $(\gamma)$, which is scaled to the surface area of the growing nucleus, combined with a volumetric free-energy difference between perfect solid and liquid $\left(g^{s l}\right)$. When nuclei are spherical,

$$
\Delta G=\frac{4}{3} \pi r^{3} g^{s l}+4 \pi r^{2} \gamma .
$$

This description sees $\Delta G$ increase with nucleus radius $(r)$ to a peak at some critical size, above which the probability of further growth increases exponentially. A critical size exists for each supercooled temperature where the value of $\Delta G$ defines the probability of formation and therefore $\tau_{w}$. The form of $\Delta G$ predicts the conditions under which freezing will occur spontaneously within a homogeneous liquid. In heterogeneous nucleation $\Delta G$ is reduced by a preexisting site lessening the penalty of a solid-liquid interface. 
CNT has been found to successfully describe nucleation in many cases (e.g., hard-sphere colloids [5] and water [6]); however, this simple representation of the nucleation process is not expected to predict the behavior of complex systems such as polymers and enzymes (e.g., [7]). For example, behavior where the initially nucleating phase differs from the critical nucleus is neglected [8]. Stranski and Totomanow [9] suggested that the nucleating phase is not the most stable, but is instead the phase with the smallest $\Delta G$, contrasting the assumption made in CNT where the difference in free energies is that between the most stable solid and the liquid (e.g., [4]). In simple liquids [10] and face-centered cubic (fcc) stable metals [11-13] it has been shown that there is a preference for body-centered cubic (bcc) arrangements to nucleate despite other phases being more stable. Nonclassical nucleation is then required to describe this kind of behavior (e.g., [7]). Here we will constrain the applicability of CNT to the Earth's core and examine whether it can sufficiently describe the nucleation process to be useful in resolving the inner core nucleation paradox.

It is not always possible to observe the freezing of a system despite undercooling. CNT predicts that the average duration before a supercooled system undergoes freezing varies exponentially with $T$. The Earth's core has cooled at $50-150 \mathrm{~K} \mathrm{Gyr}^{-1}$ [14-18] and thus crystallization of the inner core must have occurred at relatively small undercooling and therefore with large $\tau_{w}\left(\sim 10^{32} \mathrm{sm}^{-3}\right)$. The timescales relevant here are clearly not practicable to experiment or simulation. Where long waiting times exist, such as with the inner core nucleation paradox, one of two approaches is typically employed when using CNT. First, known thermodynamic properties can be used to estimate $\Delta G$ and $I_{0}$ and predict the relationship between undercooling and waiting time. We call this the thermodynamic estimate, which was used by Huguet et al. [4]. This requires explicit knowledge of free energies of both phases and the interface between them, all of which are nontrivial to obtain and often only accessible to theoretical studies (e.g., [19]). Alternatively, freezing can be observed directly in simulations at far larger undercooling; this provides waiting times for which CNT is used as a fitting model. Observed $\tau_{w}$ are then fitted with thermodynamic quantities being free parameters; we call this direct simulation. Equation (1) is then used with these properties to extrapolate to the conditions under study (e.g., [20]). Herein lies great difficulty as waiting time increases exponentially with temperature; Davies et al. [20] extrapolate observed waiting times over approximately 60 orders of magnitude. The advantage of direct simulation over the thermodynamic estimate is that no assumption need be made about some of the more uncertain thermodynamic quantities such as interfacial energy, although an assumption of nucleating phase is typically still applied. Both Huguet et al. [4] and Davies et al. [20] assume $g^{\text {sl }}$ to be represented by liquid iron and hexagonal-close-packed (hcp) iron, while the latter use $\gamma$ and $I_{0}$ to fit observed waiting times. Additionally, both assume the individual components of CNT to well represent the nucleation of iron at extreme pressure and temperature.

In this study we apply an approach to testing the application of CNT to the Earth's core, circumventing the disadvantages of both direct simulation and thermodynamic estimate approaches. We use previously developed techniques to identify subcritical nuclei in the supercooled liquid and use CNT to describe their distributions. CNT then predicts critical nuclei from these distributions and allows the calculation of all necessary thermodynamic quantities without observing freezing. We therefore test the description of $I_{0}$ and $\Delta G$ within CNT and their ability to describe nucleation in the Earth's core. The efficiency of this approach allows temperatures close to the melting point to be characterized, removing the need for extrapolation. Crucially, we make no assumption of the nucleating phase, a limitation of CNT and the previous attempts to resolve the nucleation paradox.

\section{METHODS}

$I$ (which has units of per unit time per unit volume) is recorded for all observed nucleus sizes at temperatures below the melting point of iron at $320 \mathrm{GPa}$. These distributions predict the critical radii $r_{c} . r_{c}$ is defined in CNT by $\gamma$ (which has small temperature dependence) and $g^{s l}$ via Eq. (2) where $\frac{d \Delta G}{d r}=0$, meaning that the distribution of nuclei characterizes the thermodynamics of the system. $I_{0}$ and the Zeldovich factor (z) can also be extracted from molecular dynamics information of prefreezing nuclei and so $\tau_{w}$, defined as

$$
\tau_{w}=\tau_{0} \exp \left(\frac{\Delta G(r)}{k_{B} T}\right),
$$

where $\tau_{0}=\frac{z}{I_{0}}$, can be estimated for temperatures below the melting point without the need for freezing events to be observed. As such, little extrapolation is needed to predict behavior at small undercooling.

Calculations are performed using the Large-scale Atomic/Molecular Massively Parallel Simulator [21]. The embedded atom model of iron by Alfè et al. [22] is applied with a cut-off distance of $5.5 \AA$ A. 6912 atoms are used, with a face-centered-cubic initial configuration $(12 \times 12 \times 12$ unit cells) and volume is varied to maintain $320 \mathrm{GPa}$ in the liquid at each temperature. A 6912-atom system is found to be suitably large, given reproduction of liquid structure, pressure, and energy within error of a 40 000-atom system. Furthermore, Davies et al. [20] show that this same comparison of system sizes produces near identical average waiting times to observe freezing. Liquid initial conditions are set by randomly prescribing initial velocities corresponding to a kinetic energy of $10000 \mathrm{~K}$ within the NVT ensemble. This is thermally equilibrated for 10 ps before cooling to a target temperature over $1 \mathrm{ps}$, followed by a minimum observation time of $1 \mathrm{~ns}$ (provided freezing does not occur). The simulation time step is $1 \mathrm{fs}$ and atomic positions are recorded every 100 steps. Calculations are performed in the NVE ensemble as the onset of freezing produces a significant temperature rise in the system under constant energy conditions. This provides a marker for the freezing of the system independent of structural analysis and a direct comparison to the results of Davies et al. [20].

Separate challenges exist for examining large and small undercoolings. At small undercooling, nucleation events are less common; we use long observation times to offset this. For large undercooling, nucleation events are more common; however, successful nucleation events (freezing the system) 

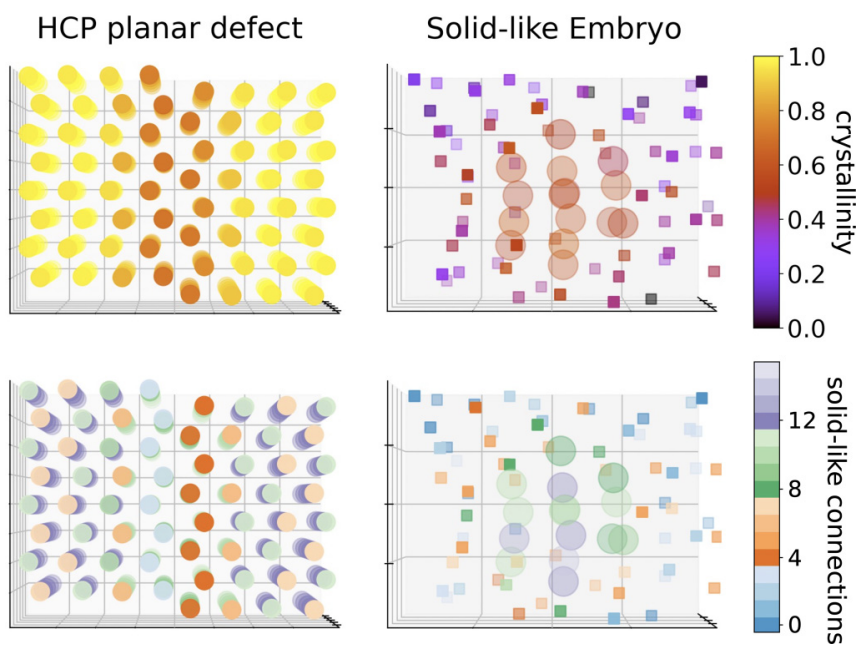

FIG. 1. Per-atom values of crystallinity (structure correlation, upper panels) and number of correlated neighbors (lower panels) for hcp Fe with a planar defect in the $Y$ plane (left) and an approximately spherical solidlike nucleus of 14 atoms surrounded by liquid (right, circles and squares, respectively). Grid spacing is $2.5 \AA$.

are also more common and limit the duration of observation. In order to collect suitable statistics, 100 unique trajectories per temperature are collected between 4800 and $5800 \mathrm{~K}$. Between 4100 and $4600 \mathrm{~K}, 40$ trajectories are found to be sufficient to predict a critical radius consistent with the overall temperature behavior, albeit with larger uncertainty than temperatures with more observed nuclei due to overall longer aggregate observation time.

Prefreezing nuclei can be defined as collections of atoms that exhibit solidlike behavior. Here the categorizing behavior is that the bonding environment of an atom is similar to that of solid iron and that this configuration is strongly correlated with that of neighboring atoms, meaning the structure is not limited to a single atom. We follow a previously developed method whereby spherical harmonics are used to categorize bonding environments surrounding each atom [23-25]. A suite of spherical harmonics is selected which construct local order parameters around atoms giving a measure of crystallinity which describes the distribution of atoms around a central atom in terms of similarity to a solid bonding configuration (see Supplemental Material for details of solidlike behavior identification [26]). In order for this to be effective, the local order parameters are tuned to give a positive response to bonding environments consistent with all phases of iron relevant to the core (bcc, fcc, and hcp) without strongly favoring or overlooking any one specifically. Crystallinity does not solely characterize a solidlike particle. When a threshold number of eight neighboring atoms all have a crystallinity $\geqslant 0.5$, the central atom is considered to be confined within a solid structure and is defined as solidlike. A criteria of eight neighbors is chosen as we find that pure liquids rarely see eight or more of these connections per atom (consistent with previous studies, e.g., [24]). The solid phases should have some number of connections below the coordination number of the perfect crystal due to thermal vibrations. Figure 1 shows examples of crystallinity and connections for a defect and a nucleus. In the example of a planar defect, all atoms remain strongly crystalline but the disruption in connections forms a discontinuity in the solidlike structure. In the case of a solidlike nucleus, solidlike atoms in isolation do not constitute a nucleus. Within each snapshot solidlike atoms are identified and those within bonding distance (defined by the solid radial distribution function) are considered to belong to the same nucleus. Nuclei sharing greater than half of the same unique atoms in adjacent snapshots are determined to be the same nucleus.

Removing terms that are constant at fixed temperature from Eqs. (1) and (2) we can describe a proportional form of the free-energy barrier:

$$
-\ln \left[I_{T}(r)\right] \propto \Delta G_{T}(r) .
$$

This allows us to use the distribution of observed nuclei sizes to produce a representation of the free energy associated with forming each nucleus size. We find that nuclei with fewer than ten atoms force nonspherical geometry, but all nuclei are pseudospherical with sphericity increasing with size and therefore find a spherical form to the scaling of $g^{s l}$ and $\gamma$ is necessary. This single temperature distribution is then

$$
\Delta G_{T}(r)=4 / 3 \pi r^{3} A+4 \pi r^{2} B,
$$

where $A$ and $B$ are fit to simulation data to estimate the radius corresponding to the peak of the free-energy barrier

$$
r_{c}=-2 B / A \text {. }
$$

Care must be taken in applying Eqs. (5) and (6). In order for a well represented distribution of nucleation rates to be collected, the simulation must run for a considerable time, exponential to temperature, where freezing presents a limit to the observation of $I$. Clearly, without freezing being observed, nuclei at or beyond the peak of $\Delta G$ will be scarce (the probability of a nucleus growing increases exponentially above the critical size making their observation without freezing unlikely) and so the fitting can only be applied to the distribution of nuclei smaller than the critical radius (see inset of Fig. 2). We are able to predict $r_{c}$ up to $5800 \mathrm{~K}$ (reported in Fig. 2) above which the form of nucleation rate is too poorly represented to be robustly fit with the form of Eq. (5) given number, size, and duration of simulations in this study.

Applying this methodology to the aggregate of all simulation distributions, we construct $r_{c}$ at each temperature (points in Fig. 2) but not the temperature dependence, to avoid a requirement of $I_{0}$. These $r_{c}$ are fitted via $g^{s l}$ and $\gamma$ where for pure iron

$$
g^{s l} \approx h_{f} \frac{\delta T}{T_{m}}\left(1-h_{c} \delta T\right)
$$

approximates the temperature dependence of $g^{s l}$. $h_{f}$ is the enthalpy of fusion and $h_{c}$ is a correction to account for the nonlinear behavior of $g^{s l}$, which has been found necessary for iron at these conditions [20]. $\delta T$ is undercooling relative to the melting temperature, $T_{m}$, set at $6215 \mathrm{~K}$ for $320 \mathrm{GPa}$ following Alfè $e t a l$. [27]. $r_{c}$ then varies with temperature as

$$
r_{c}(T)=\frac{-2 \gamma}{h_{f} \frac{\delta T}{T_{m}}\left(1-h_{c} \delta T\right)} .
$$




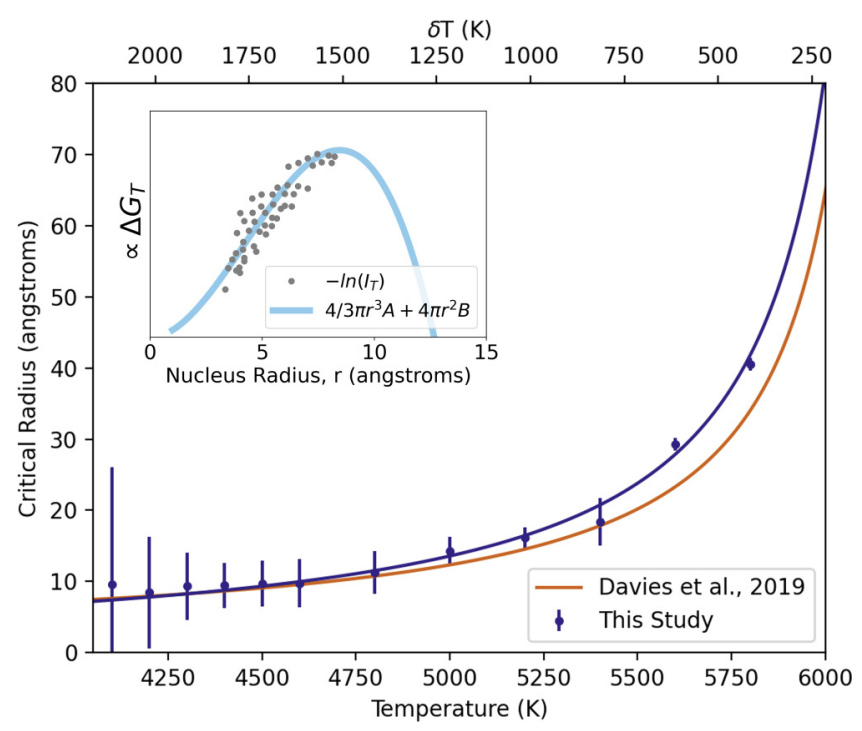

FIG. 2. Critical radii of prefreezing nuclei with temperature. Estimates of critical radius from nucleation rate are calculated at each temperature (purple circles) via Eq. (6) where $A$ and $B$ are only used for these points and absolute values of $\propto \Delta G_{T}$ are not meaningful without inclusion of $I_{0}$. Temperature dependence of critical radii is fitted with $h_{f}, h_{c}$, and $\gamma$ as free parameters via Eq. (8) (purple line) and compared to the prediction from direct simulation [20] (orange line). Increasing uncertainty with undercooling is due to less recorded nuclei, an intractable combination of fewer simulations and freezing events halting observation. The effect on statistical uncertainty of fewer nucleation events at higher $T$ is more than compensated by the long observation periods. Inset: fitting of an example nucleation rate distribution at $4200 \mathrm{~K}$ and $320 \mathrm{GPa}$.

We assume interfacial energy to be constant with temperature, a standard assumption in classical and nonclassical nucleation theory (e.g., [1] and [7]) that is confirmed by our results (see Sec. III). We fit $\gamma$ to observed waiting times using Eqs. (3), (2), and (7). $h_{f}$ and $h_{c}$ are treated as free parameters in defining $r_{c}(T)$ (Fig. 2). This allows us to fit the predicted critical radius from molecular dynamics to describe $g^{s l}$ with no assumption of the phases involved.

The kinetic prefactor (e.g., [28]) can be defined by

$$
\tau_{0}=\frac{z}{N S}
$$

where $N$ is the number of available nucleation sites, $S$ is the rate at which atoms are attached to nuclei, and $z$ relates the rate of growth to the principle that clusters have some probability of shrinking having grown to a given size. In nucleation theory $z$ is known as the Zeldovich factor and is a dimensionless quantity taken from the second derivative of free energy at the top of the free-energy barrier

$$
z=\left(\frac{\frac{4}{3} \pi r_{c}^{3} g^{s l}}{k_{B} T}\right)^{-1 / 2}
$$

We calculate $N$ as the average number of nuclei of any size present at any one snapshot and $S$ as the average growth rate of nuclei between snapshots.
TABLE I. Thermodynamic quantities required to calculate waiting times [Eq. (3)] from nucleation rates in this study, compared to those used in the thermodynamic estimate and direct simulation methods.

\begin{tabular}{lcccc}
\hline \hline Name & Units & This study & $\begin{array}{c}\text { Thermodynamic } \\
\text { estimate }\end{array}$ & $\begin{array}{c}\text { Direct } \\
\text { simulation }^{\mathrm{b}}\end{array}$ \\
\hline$\tau_{0}$ & $\mathrm{~s} \mathrm{~m}^{-3}$ & $5.742 \times 10^{44}$ & $5 \times 10^{39}$ & $7.04 \times 10^{47}$ \\
$h_{f}$ & $\mathrm{~J} \mathrm{~m}^{-3}$ & $7.119 \times 10^{10}$ & $1 \times 10^{10}$ & $0.98 \times 10^{10}$ \\
$h_{c}$ & & $6.609 \times 10^{-5}$ & 1 & $7.05 \times 10^{-5}$ \\
$\gamma$ & $\mathrm{J} \mathrm{m}^{-2}$ & 1.02065 & 1.2 & 1.08 \\
$\delta T_{\text {core }}$ & $\mathrm{K}$ & 807 & 1000 & 730 \\
\hline \hline
\end{tabular}

${ }^{\mathrm{a}}$ Huguet et al. [4].

${ }^{\mathrm{b}}$ Davies et al. [20].

\section{RESULTS}

$r_{c}$ is found to decrease with increasing undercooling, a key prediction of CNT and direct validation of the expected nucleation behavior of a supercooled liquid. The change in $r_{c}$ with temperature agrees well with the prediction of Davies et al. [20], especially at large undercooling where that study's observations are made (see Fig. 2). At low undercooling (temperatures greater than $4600 \mathrm{~K}$ ), the critical radius is larger than the previous prediction (26\% larger than Davies et al. [20] at $6000 \mathrm{~K}$ ) but is still captured well by the formalism of CNT.

We examine the assumption applied in CNT that interfacial energy is a temperature invariant quantity. Using free energies of solid and liquid to estimate $g^{s l}$ independently, we calculate $\gamma$ for each prediction of $r_{c}$. The solid free energy is taken as hcp Fe from Alfè et al. [29] and the liquid value is obtained by extrapolation from the melting curve of Alfè et al. [27] (where $G^{l}=G^{s}$ ) using thermodynamic properties from Ichikawa et al. [30]. We find that $r_{c}(T)$ accommodates a maximum of $10^{-4} \mathrm{~J} \mathrm{~m}^{-2} \mathrm{~K}^{-1}$ gradient of $\gamma$, while the mean value of $\gamma$ is $1.42 \mathrm{~J} \mathrm{~m}^{-2}$. The single value of $\gamma$ which produces the best fit to observed waiting times and all predicted $r_{c}$ is $1.02065 \mathrm{~J} \mathrm{~m}^{-2}$, slightly smaller than that found by previous works $[19,20]$. Enthalpy of fusion is $7.119 \times 10^{9} \mathrm{~J} \mathrm{~m}^{-3}$ with a temperature dependence of $6.609 \times 10^{-5}$ resulting in a smaller value of $g^{s l}$ at all temperatures when compared to the previous studies (18\% and 28\% less than Huguet et al. [4] and Davies et al. [20]; see Supplemental Material for details of free-energy differences [26]). All values and comparisons are shown in Table I.

$\tau_{0}$ is found to vary little with temperature due to a compensatory effect of $N$ and $z$ with $S$ (see Supplemental Material for details of temperature dependence of $\tau_{0}$ [26]). An average value across temperatures is $5.742 \times 1044 \mathrm{~s} \mathrm{~m}^{-3}$, significantly smaller than Davies et al. [20] $\left(10^{48}\right)$ who determined $I_{0}$ as a freely fitted parameter, and larger than Huguet et al. [4] $\left(10^{40}\right)$ who note that an exact value is of little importance due to a small temperature sensitivity compared to the free-energy barrier term (see Table I). Our value is similar to that of Christian [1] $\left(10^{42}\right)$ where the value is estimated from reasonable nucleus densities and enthalpy of fusion at observable conditions and is assumed to be mostly temperature invariant. 


\section{ठТ (K)}

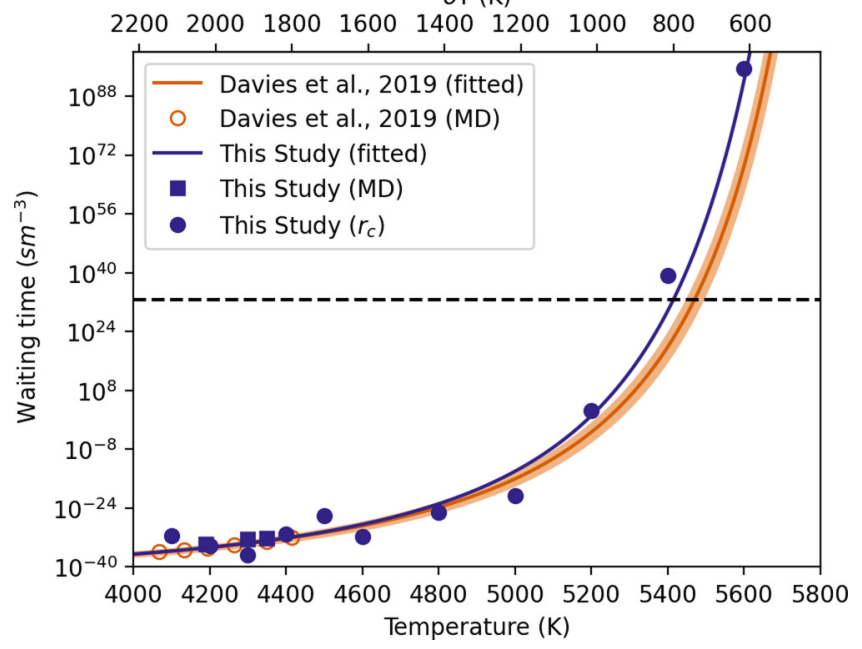

FIG. 3. Waiting times for nucleation predicted by the analysis of subcritical nuclei in supercooled liquids (this study, purple circles) is compared to direct simulations (squares and orange circles). Fitting for these two approaches is shown as lines (purple and orange, respectively) and the dashed line represents the waiting time required to freeze the present volume of the Earth's inner core given an age of 1 Gyr.

Where simulations regularly freeze (at large $\delta T$ ) we compare to direct simulation (also at $320 \mathrm{GPa}$ ) [20] and find good agreement (Fig. 3). In these cases we consistently find hcp Fe with planar defects, which eventually relax. The predicted waiting time to observe freezing (Fig. 3) is found to be consistent with Davies et al. [20] at large undercooling, and is longer at small undercooling where this work is able to sample more directly. This is due to the difference in $h_{c}$ as well as $g^{s l}$ being smaller at all temperatures. When evaluating the undercooling required to produce freezing after $4.43 \times 10^{32} \mathrm{~s} \mathrm{~m}^{-3}$ (consistent with the predicted first nucleation of the Earth's inner core at $1 \mathrm{Ga}$ [20]) we find an $807 \mathrm{~K}$ undercooling is required. This is intermediate to $730 \mathrm{~K}$ from Davies et al. [20] and $1000 \mathrm{~K}$ from Huguet et al. [4].

\section{CONCLUSION}

The distribution of prefreezing nuclei in supercooled liquids is found to accurately predict the critical radius for nucleation in liquid iron at the high pressures and temperatures relevant to the Earth's core (320 GPa, 4000-5800 K). Our method provides insight into the behavior of supercooled liquids at temperatures close to $T_{m}$ and at much smaller undercooling than is accessible to other methods which follow CNT. We test the validity of CNT through its prescription of a kinetic prefactor and free-energy barrier description of the nucleation process. We do this solely through the observation of prefreezing nuclei in molecular dynamics and find that the distribution of nuclei can predict waiting times for freezing of high pressure liquid iron in agreement with studies which take different routes to applying CNT.

The 5\% smaller value of interfacial energy compared to Davies et al. [20] can perhaps be explained by a difference in structure in small nuclei compared to the bulk solid, consistent with a smaller difference in free energies and an overall reduction in the free-energy barrier. Stranski and Totomanow [9] pose the first nucleating phase is not that with the lowest free energy, but that with the smallest free-energy barrier. In cases where simulations completely freeze, at large undercooling, we observe defects relaxing from the solid some time after a successful nucleation event. This is commensurate with a less negative value of $g^{s l}$ at all temperatures compared to other works which assume the enthalpy of fusion to be that of forming hep iron $[4,20]$. We make no assumption about the nature of the nucleating phase, nor its thermodynamic properties. Instead we find the properties that best describe the behavior of subcritical nuclei in the supercooled liquid. These properties reveal that the nucleating solid is less favorable than hcp iron and so while generally describing the system, this fundamental assumption of CNT is not valid for Earth's core, and nonclassical nucleation theory provides a more appropriate description. The structure of nucleating material we observe is best described as defect-rich hcp; however, a detailed structural analysis is beyond the scope of this study.

We compare estimates of undercooling required to freeze in a system (for a given waiting time) with studies which apply CNT through thermodynamic estimate and direct simulation methods. The key assumption made in this work is simply that the energy of small nuclei is representative of critical nuclei, very different to those made by previous works; however, we arrive at a similar prediction of waiting times. An undercooling of $807 \mathrm{~K}$ is found to be intermediate to previous works, where the waiting time is related to the time required to produce the Earth's solid inner core. While the inner core nucleation paradox remains unresolved through the study of a pure iron system, the study of prefreezing nucleation gives access to undercoolings which were previously unattainable and describes the thermodynamic properties of nucleating systems which must otherwise be assumed. This provides a framework for examining nucleation in impure systems at core conditions, towards resolving the inner core nucleation paradox.

\section{ACKNOWLEDGMENTS}

We acknowledge a Natural Environment Research Council grant, reference NE/T000228/1, which supports this project. Calculations were performed on the UK National supercomputing service ARCHER (via allocation through the Mineral Physics Consortium), ARC4 research computing facility (University of Leeds), and University College London (UCL) Research Computing facilities.
[1] J. W. Christian, The Theory of Transformations in Metals and Alloys (Pergamon Press, Oxford, 2002).
[2] R. Sharma and G. Purdy, Nucleation limitation and hardenability, Metall. Trans. 5, 939 (1974). 
[3] W. Cantrell and A. Heymsfield, Production of ice in tropospheric clouds: A review, Bull. Am. Meteorol. Soc. 86, 795 (2005).

[4] L. Huguet, J. A. Van Orman, S. A. Hauck II, and M. A. Willard, Earth's inner core nucleation paradox, Earth Planet. Sci. Lett. 487, 9 (2018).

[5] S. Auer and D. Frenkel, Numerical prediction of absolute crystallization rates in hard-sphere colloids, J. Chem. Phys. 120, 3015 (2004).

[6] R. C. Miller, R. J. Anderson, J. Kassner, Jr., and D. E. Hagen, Homogeneous nucleation rate measurements for water over a wide range of temperature and nucleation rate, J. Chem. Phys. 78, 3204 (1983).

[7] S. Karthika, T. Radhakrishnan, and P. Kalaichelvi, A review of classical and nonclassical nucleation theories, Cryst. Growth Des. 16, 6663 (2016).

[8] I. Ford, Nucleation theorems, the statistical mechanics of molecular clusters, and a revision of classical nucleation theory, Phys. Rev. E 56, 5615 (1997).

[9] I. Stranski and D. Totomanow, Nucleation rate and OstWald's step rule, Mag. J. Phys. Chem. 163, 399 (1933).

[10] W. Klein and F. Leyvraz, Crystalline Nucleation in Deeply Quenched Liquids, Phys. Rev. Lett. 57, 2845 (1986).

[11] R. E. Cech, Evidence for solidification of a metastable phase in Fe-Ni alloys, Trans. Am. Inst. Min. Metall. Eng. 206, 585 (1956)

[12] Y.-W. Kim, H.-M. Lin, and T. F. Kelly, Solidification structures in submicron spheres of iron-nickel: Experimental observations, Acta Metall. 36, 2525 (1988).

[13] W. Löser, T. Volkmann, and D. Herlach, Nucleation and metastable phase formation in undercooled $\mathrm{Fe}-\mathrm{Cr}-\mathrm{Ni}$ melts, Mater. Sci. Eng., A 178, 163 (1994).

[14] A. Souriau, Deep earth structure-The earth's cores, Treatise on Geophysics, 1, Seismology and Structure of the Earth (Elsevier, New York, 2007), Chap. 19, pp. 655-693.

[15] F. Nimmo, Energetics of the core, in Treatise on Geophysics, 2nd ed., edited by G. Schubert (Elsevier, Amsterdam, 2015), Vol. 8, pp. 27-55.

[16] R. Deguen, Structure and dynamics of Earth's inner core, Earth Planet. Sci. Lett. 333-334, 211 (2012).

[17] D. J. Stevenson, Mars' core and magnetism, Nature (London) 412, 214 (2001).
[18] C. Davies, M. Pozzo, D. Gubbins, and D. Alfè, Constraints from material properties on the dynamics and evolution of Earth's core, Nat. Geosci. 8, 678 (2015).

[19] W.-J. Zhang, Z.-Y. Liu, Z.-L. Liu, and L.-C. Cai, Melting curves and entropy of melting of iron under Earth's core conditions, Phys. Earth Planet. Inter. 244, 69 (2015).

[20] C. J. Davies, M. Pozzo, and D. Alfè, Assessing the inner core nucleation paradox with atomic-scale simulations, Earth Planet. Sci. Lett. 507, 1 (2019).

[21] S. Plimpton, Fast parallel algorithms for short-range molecular dynamics, J. Comput. Phys. 117, 1 (1995).

[22] D. Alfè, M. Gillan, and G. Price, Complementary approaches to the $a b$ initio calculation of melting properties, J. Chem. Phys. 116, 6170 (2002).

[23] P. Rein ten Wolde, M. J. Ruiz-Montero, and D. Frenkel, Numerical calculation of the rate of crystal nucleation in a Lennard-Jones system at moderate undercooling, J. Chem. Phys. 104, 9932 (1996).

[24] J. Persson, C. Desgranges, and J. Delhommelle, Polymorph selection during the crystallization of iron under the conditions of Earth's inner core, Chem. Phys. Lett. 511, 57 (2011).

[25] J. Van Duijneveldt and D. Frenkel, Computer simulation study of free energy barriers in crystal nucleation, J. Chem. Phys. 96, 4655 (1992).

[26] See Supplemental Material at http://link.aps.org/supplemental/ 10.1103/PhysRevB.103.214113 for details on identification of solidlike behavior, solid-liquid free-energy difference, and temperature dependence of the kinetic prefactor.

[27] D. Alfè, G. D. Price, and M. J. Gillan, Iron under Earth's core conditions: Liquid-state thermodynamics and high-pressure melting curve from $a b$ initio calculations, Phys. Rev. B 65, 165118 (2002).

[28] R. P. Sear, Nucleation: Theory and applications to protein solutions and colloidal suspensions, J. Phys.: Condens. Matter 19, 033101 (2007).

[29] D. Alfè, G. D. Price, and M. J. Gillan, Thermodynamics of hexagonal-close-packed iron under Earth's core conditions, Phys. Rev. B 64, 045123 (2001).

[30] H. Ichikawa, T. Tsuchiya, and Y. Tange, The $P-V-T$ equation of state and thermodynamic properties of liquid iron, J. Geophys. Res.: Solid Earth 119, 240 (2014). 